\title{
Outage and Rate Evaluation of Drone based Decode and Forward Cooperation for Hybrid Fading Channels
}

\author{
Nikita Goel and Vrinda Gupta, Member, IEEE
}

\begin{abstract}
In this paper, we consider a drone as a relay in Cooperative Communication (CC) to improve the network performance in an upcoming wireless network. Drone Assisted CC (DA$\mathrm{CC}$ ) is more useful when the central coordinator (base station) gets disrupted. In such a scenario, the drone works as an aerial relay and provides $\mathrm{CC}$ diversity to the end-users. In this article, a Decode-and-Forward (DF) protocol is used as a relaying scheme at the drone, and the Maximal Ratio Combining (MRC) scheme is used at the end-users for combining the direct and relayed signal. Here, we assume Nakagami faded channel among Airto-Ground (A2G) links and Rayleigh faded distribution between Ground-to-Ground (G2G) links. The performance of DA-CC is evaluated in a hybrid channel environment and compared based on drone height, rate, horizontal distance, and transmitted power with the existing Rayleigh and Nakagami faded distributions. The analytical expression of outage probability and the rate have been derived for analysis purposes, and Monte-Carlo simulations are used to verify the analytical results. This work can have security and surveillance applications to improve the network performance in the absence of a central base station.
\end{abstract}

\section{INTRODUCTION}

Cooperative Communications (CC) meets the high data rate requirements of $5 \mathrm{G}$ wireless communications without increasing bandwidth or power by enhancing system capacity and coverage [1]. Through developing virtual multiple antenna connectivity environments, $\mathrm{CC}$ solves the effect of multipath fading by enabling cooperative diversity [1], [2]. The incorporation of a $\mathrm{CC}$ relay channel provides separate pathways between the source and the end-users, and it is believed that each wireless user (source) transmits data and supports another by using a cooperation strategy.

Different relaying schemes are used in $\mathrm{CC}$ for forwarding the messages named as Amplify-and-Forward (AF), Decodeand-Forward (DF), etc. as explained by [2], [3]. Here we are using DF as a relaying scheme for analysis purposes.

Manuscript received December 4, 2020; revised May 11, 2021. Date of publication June 16, 2021. Date of current version June 16, 2021. The associate editor prof. Francesca Vatta has been coordinating the review of this manuscript and approved it for publication.

$\mathrm{N}$. Goel is pursuing PhD with the Department of Electronics and Communication Engineering, National Institute of Technology Kurukshetra, India. Currently she is working as an Assistant Professor in the Department of Electronics and Communication Engineering, K.I.E.T Group of Institutions Delhi NCR Ghaziabad, India (e-mail: nicks.miet@gmail.com).

V. Gupta is with the Department of Electronics and Communication Engineering, National Institute of Technology Kurukshetra, India (e-mail: vrindag16@gmail.com).

Digital Object Identifier (DOI): 10.24138/jcomss-2020-0013
There are several sites, for example, unintended natural disaster zones, congested areas, mountain areas, or homes, where conventional cooperative networks are unable to provide Quality of Service (QoS) due to transmitter-receiver blockage or even a total lack of communication may occur [4]. An alternative link through relay networks consisting of drones that act as message forwarding nodes supporting transmitterreceiver communication is considered a possible solution to this problem [5], [6].

Many researchers have studied CC using Unmanned Aerial Vehicle (UAV)/drone as a relay. In [7], a dual-hop Cooperative Relaying Strategy (CRS) for Non-orthogonal Multiple Access (NOMA) under Rayleigh fading has been proposed, and results are compared with the conventional scheme. The researchers have proposed a new opportunistic two-way relaying method where sources are allowed to transmit without using distributed space-time coding [8]. Performance analysis of the hybrid relaying scheme is investigated in [9] and compared with the existing adaptive relaying scheme. In a similar way, authors in [10] have derived Symbol Error Probability (SEP) by considering hybrid decode amplify and forward relaying scheme. Ghofrani-Jahromi et al. [11] have derived outage probability, diversity order, and gain of the system in Nakagami faded environment.

The Symbol Error Rate (SER) is calculated in Nakagami faded environment using DF relaying scheme in [12]. Considering the Nakagami fading environment, performance analysis of multiple dual-hop DF relay networks appears as mentioned in [13]. The analysis of the outage probability of dual-hop threshold DF-based cooperative system for different relay selection schemes is presented in [14]. A hybrid technique is described in [15], to reduce the effects of Relay Node $(\mathrm{RN})$ induced error propagation, considering both $\mathrm{RN}$ location and RN-Bit Error Ratio (BER). In [16], under the Multiple Input Single Output (MISO) scenario, One Way Relay Channel (OWRC) has been studied. Outage probability and error rate are derived in [17] using AF relaying scheme in Nakagami faded environment. A comprehensive survey of all developments promoting smooth integration of UAVs into cellular networks has been presented in [18]. Also, it provides a detailed insight into the type of consumer UAVs, interference issues, their solutions, challenges, opportunities of UAV-based relay communication systems and physical security issues of UAV-assisted systems. 
Bin Li et al. [19] have provided a brief understanding of 5G wireless networks' UAV communications and launched advanced space-air-ground networks. Various challenges faced by the system have been discussed in this paper. The expression of optimal drone height is evaluated in [20] without considering the G2G link into consideration. In [21], the DFbased CC scenario under different channel conditions have been analyzed. The authors proposed a power allocation algorithm when all nodes are on the same horizon plane. A study of the best relay selection technique in various cooperative relaying schemes has been given in [22]. It explores how $\mathrm{CC}$ overcomes the challenges faced in $5 \mathrm{G}$ communications by millimeter-wave. The authors in [23] evaluated system performance using AF as a relaying scheme and MRC. Further Selection Combining (SC) has been taken for combining the signals at the destination. Besides, the Weibull channel is chosen for the source to relay and the source to the destination connection, and the Rician channel for relay to the destination link. Drone-based network coded cooperative communication has been analyzed in [24]. In [25], researchers have proposed a novel drone assisted network coded cooperation and analysed network coded noise expression. Multiple Input Multiple Output (MIMO) drone based cooperative retransmission has been discussed in [26]. The authors of [27] have implemented a multiple drone system that consists of a quadcopter and demonstrate it in rescue operations. In [28], DA-CC performance assuming AF as relaying protocol has been evaluated. Furthermore, in this work, the performance in the hybrid channel environment has been analyzed, and rate equations for different channel environments have been derived. However, mathematical modeling for the same has not been provided.

This research paper provides a context for evaluating the performance of DA-CC systems for DF relaying protocol over hybrid fading networks, unlike previous study from [28]. A hybrid channel is considered a more practical scenario. Therefore this research work addresses the system performance in this environment. Considering Nakagami and Rayleigh fading, this study extracts the closed-form approximate expression of the outage over hybrid fading channels for analysis. The rate equation for DA-CC in different channel environments is also given. With simulation outcomes, all analytical derived expressions are confirmed successfully.

Our paper's main contributions are summarized as follows:

- Unlike previously published work [28], we have analyzed the performance of the CC system considering DF as a relaying scheme and using a drone as a relay which happens to be more realistic hybrid fading climate. We have considered three separate cases for analysis purposes.

(i) All the links are faded from Rayleigh fading.

(ii) $S-R$ and $R-D$ links are Nakagami faded, while $S-D$ link is Rayleigh faded (proposed hybrid channel environment).

(iii) All the links have been faded from Nakagami fading.

- We have derived and provided mathematical modeling of the system's rate equation for all the above-defined cases and verified the same with simulation results.
- Also, we have obtained the expression of the system outage probability for various fading conditions and verified mathematical findings with simulation results.

The paper's remainder is listed as follows. Section 2 introduces the DA-CC system model, where the drone acts as a relay node, and the DF is used as a relaying scheme. In section 3 , outage probability and rate equations for hybrid channel conditions are derived. In section 4 , theoretical and simulated observations are drawn and analyzed. Section 5 concludes the findings of this study.

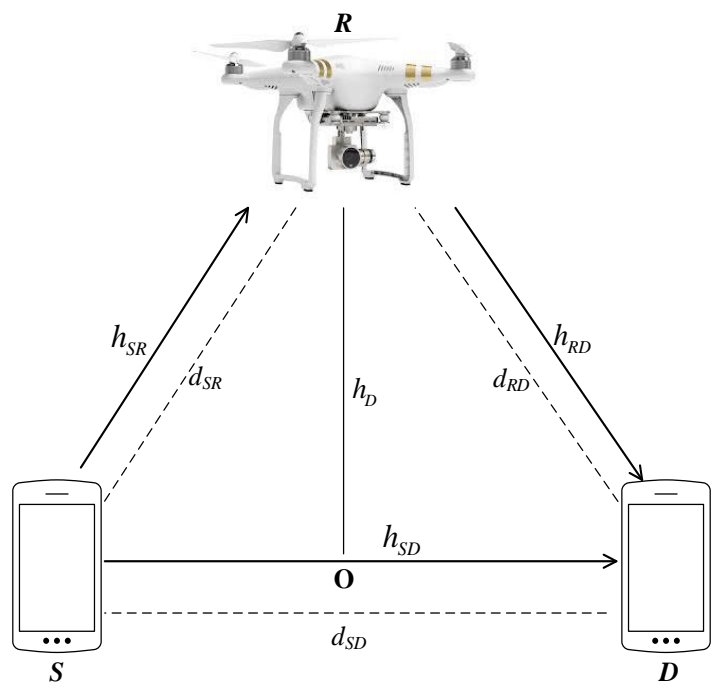

Fig. 1. System model for drone assisted cooperative communication.

\section{SySTEM MODEL}

Fig.1 illustrates the proposed DA-CC network's system model, which includes a source, destination, and relay node represented as $S, D$, and $R$ nodes, respectively. The drone $(R)$ is deployed as a relay node to facilitate diversity between the $S$ and $D$ nodes. Fig. 1 indicates that in the first time slot, $S$ transmits the data to $R$ and $D$ nodes. In the second time slot, the relay fully decodes the received data from $S$ and sends them to node $D$. We assume each node is equipped with one antenna and it transmits the signal in half-duplex mode. According to the coordinate system, the location of the $S$ and $D$ can be denoted by $\left[\begin{array}{lll}x_{s} & y_{s} & 0\end{array}\right]$ and $\left[\begin{array}{lll}x_{d} & y_{d} & 0\end{array}\right]$, respectively. The drone is assumed at fixed height $h_{D}$, the location of which is denoted by $\left[\begin{array}{lll}x_{r} & y_{r} & h_{D}\end{array}\right]$. Channel State Information (CSI) is known by a receiver node $(R, D)$ only. However, the energy-related problems of UAVs are not considered here. The symbols used in this paper are summarised in Table I.

The transmission and reception scheme for DA-CC takes two time slots. In the first time slot, $S$ transmits the signal to $D$, which is overheard by the $R$ node. The signal received at $D$ and $R$ nodes in the first time slot is given as [6]:

$$
\begin{aligned}
Y_{S D}^{\xi_{1}} & =\sqrt{P_{s} d_{S D}^{-\alpha}} h_{S D}^{\xi_{1}} x+n_{D}, \\
Y_{S R}^{\xi_{2}} & =\sqrt{P_{s} d_{S R}^{-\alpha}} h_{S R}^{\xi_{2}} x+n_{R},
\end{aligned}
$$


TABLE I

LIST OF NOTATIONS

\begin{tabular}{|l|l|}
\hline Symbol & Description \\
\hline$h_{D}$ & Height of the Drone from the ground level \\
\hline$Y_{a b}$ & Signal obtained by node $b^{t h}$ from node $a^{t h}$ \\
\hline$\alpha$ & Path loss exponent \\
\hline$d_{i j}$ & Distance between $i^{t h}$ and $j^{t h}$ node \\
\hline$h_{S R}$ & Channel coefficient between source-relay link \\
\hline$h_{R D}$ & Channel coefficient between relay-destination link \\
\hline$x$ & Symbol transmitted from the source \\
\hline$n_{R}$ & Relay node noise \\
\hline$n_{D}$ & Destination node noise \\
\hline$B$ & Bandwidth \\
\hline$\sigma^{2}$ & Variance of noise \\
\hline$C_{D A-C C}$ & Rate at destination \\
\hline$P_{S}$ & Power transmitted by source node \\
\hline$P_{R}$ & Power transmitted by relay node \\
\hline$I_{D A-C C}$ & Mutual information between direct and relayed path \\
\hline$\beta$ & Power allocation factor \\
\hline$P_{\text {out }}$ & Outage probability \\
\hline$R$ & Spectral Efficiency \\
\hline$\chi$ & Power Allocation Factor \\
\hline
\end{tabular}

where $\xi_{1}, \xi_{2} \in\{$ Rayleigh(Ray), Nakagami(Nak) $\}, \alpha$ denotes the path loss exponent, $Y_{S D}^{\xi_{1}}, Y_{S R}^{\xi_{2}}$ denotes the signal received at $D$ and $R$ node while the signal is transmitted by $S$ node. $P_{S}$ represents the power transmitted by the $S$ node, $x$ denotes the message signal transmitted by node $S$, and $h_{S D}^{\xi_{1}}, h_{S R}^{\xi_{2}}$ represents the channel coefficient between $S-D$ and $S-R$ links, respectively. Additive White Gaussian Noise (AWGN) noise at node $R$ and $D$ are represented by $n_{R}$ and $n_{D}$ with zero mean and variance $\sigma_{R}^{2}$ and $\sigma_{D}^{2}$. The distance between $S-R$, $S-D$, and $R-D$ nodes is denoted by $d_{S R}, d_{S D}$ and $d_{R D}$, respectively. In this work, we assume $R$ is moving vertically either upward or downward for a fixed position of $S$ and $D$ nodes or moving horizontally between $S$ and $D$ nodes for given $h_{D}$. For simplicity, the distance between $S-R$ link and $S-D$ link is assumed to be equal and is given as:

$$
d_{R D}=d_{S R}=\sqrt{h_{D}^{2}+\left(\frac{d_{S D}}{2}\right)^{2}} .
$$

Here, $R$ fully decodes the signal received from $S$ in the first time slot using DF as relaying scheme and sends the decoded signal towards the $D$ node in the second time slot. The signal received at $D$ in the second time slot is given as:

$$
Y_{R D}^{\xi_{2}}=\sqrt{P_{R} d_{R D}^{-\alpha}} h_{R D}^{\xi_{2}} x+n_{D},
$$

where $P_{R}$ represents the relay transmitted power. This paper has considered DA-CC under different channel environments such as Rayleigh and Nakagami fading environments. For evaluating the system performance, three different cases of channel environment have been considered, which are described as follows:

Case 1: In the first case, all the links are Rayleigh distributed. Case 2: In the second case, a hybrid channel environment is considered, as shown in Fig.2. In this case, the $S$ - $D$ link is Rayleigh distributed because of assuming only the NonLine of Sight (NLoS) component is received at node $D$. We also assume $\mathrm{A} 2 \mathrm{G}$ links ( $S-R$ and $R-D$ links) to be Nakagami distributed because of the Line of Sight (LoS) component being received at receiving node $(R$ and $D)$ due to vertical movement of the drone. For simplicity, we have considered Nakagami fading with shaping parameter $(\mathrm{m}=2)$ for developing the analytical framework. In the future, it can be generalized for other values of $\mathrm{m}$.

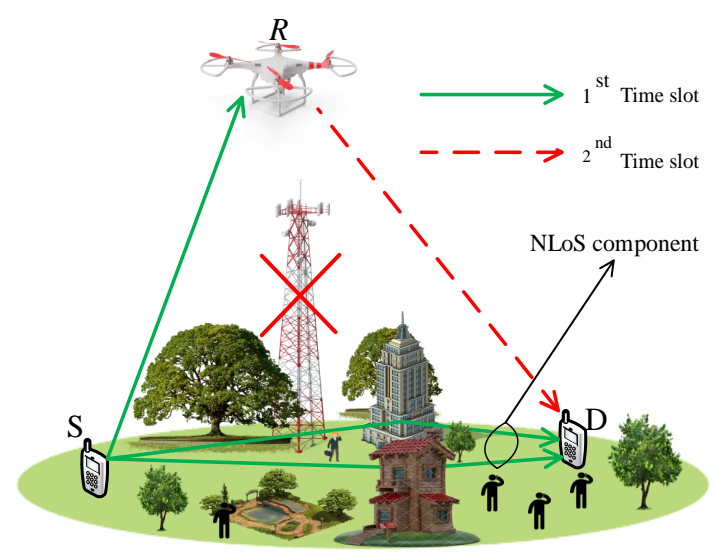

Fig. 2. Illustration of DA-CC scenario where dissimilar obstacles in $S$ and $D$ channels lead to Rayleigh fading. In particular, $S-R$ and $R$ - $D$ links are dominated by LoS components (modelled by Nakagami distribution).

Case 3: In the third case, all the links are Nakagami distributed.

The generalized power allocation scheme used in our system model is given as:

$$
\begin{array}{r}
P_{S}=\chi P_{T} \\
P_{R}=(1-\chi) P_{T},
\end{array}
$$

where $\chi$ denotes the power allocation factor and $P_{T}$ represents the power constraint which can be given as $P_{S}+P_{R}=P_{T}$.

\section{Outage And Rate Evaluation of DA-CC}

In this section, the analytical expressions for rate and outage probability are derived for evaluating the system performance under the different channel conditions.

\section{A. Rate Analysis}

Let $t$ seconds be the time needed to complete the entire process of communication in this setup, and let $\frac{t}{2}$ seconds be the time allotted to each phase. Likewise, let bandwidth available in the network be denoted by $B$ and bandwidth assigned to each time slot be $\frac{B}{2}$. Using MRC at node $D$ for combining the direct component $(S-D)$ and relayed component $(S-R-D)$ of signals under consideration, and the rate equation can be written as:

$$
\mathrm{C}_{\mathrm{DA}-\mathrm{CC}}^{\xi_{1}, \xi_{2}}=\left(\frac{B}{2}\right) \mathrm{I}_{\mathrm{DA}-\mathrm{CC}}^{\xi_{1}, \xi_{2}}
$$

where $\mathrm{I}_{\mathrm{DA}-\mathrm{CC}}^{\xi_{1}, \xi_{2}}$ denotes the mutual information between $S-D$ and $S-R-D$ path in consideration, further it can be defined as:

$\mathrm{I}_{\mathrm{DA}-\mathrm{CC}}^{\xi_{1}, \xi_{2}}=\min \left\{\log _{2}\left(1+\mathrm{SNR}_{S R}^{\xi_{2}}\right), \log _{2}\left(1+\mathrm{SNR}_{S D}^{\xi_{1}}+\mathrm{SNR}_{R D}^{\xi_{2}}\right)\right\}$, 
where $\operatorname{SNR}_{S D}^{\xi_{1}}$ denotes Signal to Noise Ratio (SNR) at node $D$ when taking $S-D$ link in consideration. It is defined as:

$$
\operatorname{SNR}_{S D}^{\xi_{1}}=\frac{P_{s} d_{S D}^{-\alpha}\left|h_{S D}^{\xi_{1}}\right|^{2}}{\sigma_{D}^{2}}
$$

where SNR at node $R$ and $D$ is denoted by $\operatorname{SNR}_{S R}^{\xi_{2}}$ and $\mathrm{SNR}_{R D}^{\xi_{2}}$ and defined as:

$$
\begin{gathered}
\operatorname{SNR}_{S R}^{\xi_{2}}=\frac{\left(P_{R} d_{S R}^{-\alpha}\left|h_{S R}^{\xi_{2}}\right|^{2}\right)}{\sigma_{R}^{2}}, \\
\operatorname{SNR}_{R D}^{\xi_{2}}=\frac{\left(P_{R} d_{R D}^{-\alpha}\left|h_{R D}^{\xi_{2}}\right|^{2}\right)}{\sigma_{D}^{2}} .
\end{gathered}
$$

Depending on $\xi_{1}$ and $\xi_{2}$, three possible cases for rate are as follows:

Case 1: In this case, all the links are considered to be Rayleigh faded, and this is shown in Fig.3. Substituting $\xi_{1}=$ Ray and $\xi_{2}=$ Ray in (8), (9), and using this in (7), the rate at destination node $D$ can be obtained as:

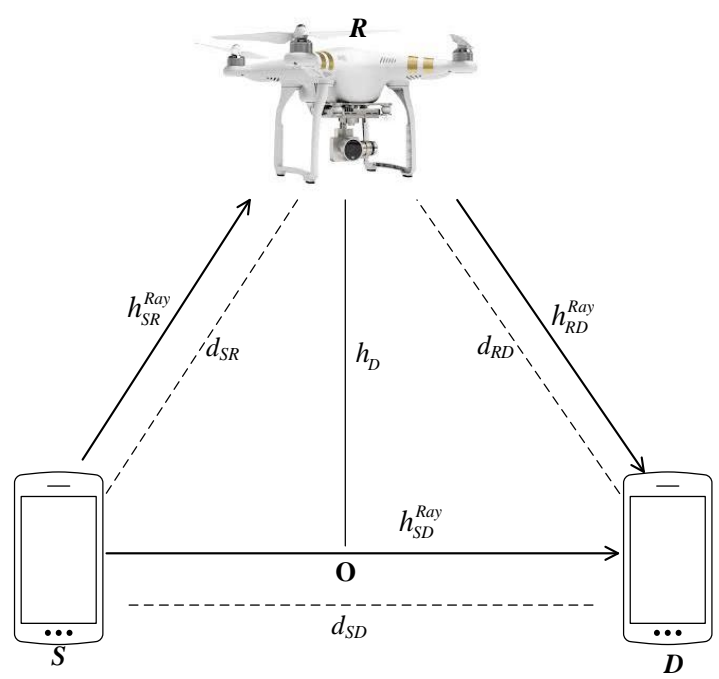

Fig. 3. All the links are Rayleigh faded.

$$
\begin{aligned}
& \mathrm{C}_{\mathrm{DA}-\mathrm{CC}}^{\text {Ray }}=\left(\frac{B}{2}\right) \min \left[\log _{2}\left(1+\frac{P_{R} d_{S R}^{-\alpha}\left|h_{S R}^{\text {Ray }}\right|^{2}}{\sigma_{R}^{2}}\right),\right. \\
&\left.\log _{2}\left(1+\frac{P_{s} d_{S D}^{-\alpha}\left|h_{S D}^{\text {Ray }}\right|^{2}}{\sigma_{D}{ }^{2}}+\frac{P_{R} d_{R D}^{-\alpha}\left|h_{R D}^{\text {Ray }}\right|^{2}}{\sigma_{D}{ }^{2}}\right)\right] .
\end{aligned}
$$

Case 2: In this case, the hybrid channel environment is under consideration. The $S-D$ link is Rayleigh faded, whereas the $S-R$ and $R-D$ links are Nakagami faded, as shown in Fig.4. Substituting $\xi_{1}=$ Ray and $\xi_{2}=$ Nak in (8) and (9), and using this in (7), the rate at $D$ can be written as shown below:

$$
\begin{gathered}
\mathrm{C}_{\text {DA-CC }}^{\text {Ray,Nak }}=\left(\frac{B}{2}\right) \min \left[\log _{2}\left(1+\frac{P_{R} d_{S R}^{-\alpha}\left|h_{S R}^{\mathrm{Nak}}\right|^{2}}{\sigma_{R}^{2}}\right),\right. \\
\left.\log _{2}\left(1+\frac{P_{s} d_{S D}^{-\alpha}\left|h_{S D}^{\mathrm{Ray}}\right|^{2}}{\sigma_{D}{ }^{2}}+\frac{P_{R} d_{R D}^{-\alpha}\left|h_{R D}^{\mathrm{Nak}}\right|^{2}}{\sigma_{D}{ }^{2}}\right)\right] .
\end{gathered}
$$

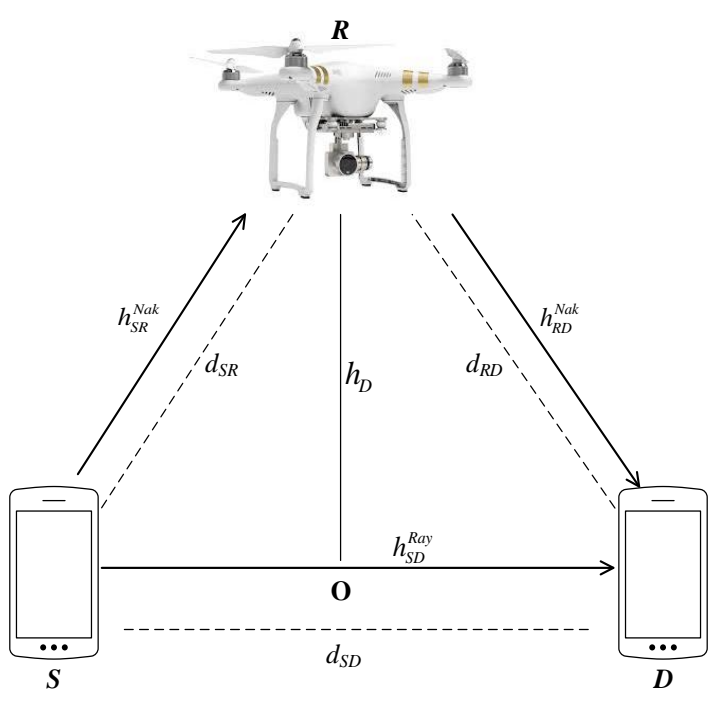

Fig. 4. Only $h_{S R}$ and $h_{R D}$ links are Nakagami faded and $h_{S D}$ is Rayleigh faded.

Case 3: Here, all the links are Nakagami faded, as shown in Fig.5. Substituting $\xi_{1}=\mathrm{Nak}$ and $\xi_{2}=\mathrm{Nak}$ in (8) and (9) and using this in (7), the rate at $D$ can be written as below:

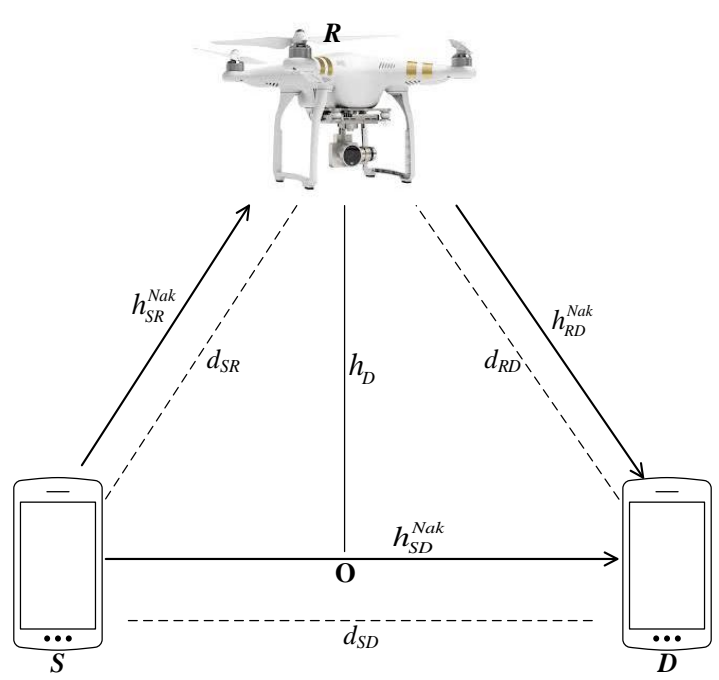

Fig. 5. All the links are Nakagami faded.

$$
\begin{gathered}
\mathrm{C}_{\mathrm{DA}-\mathrm{CC}}^{\mathrm{Nak} N \mathrm{Nak}}=\left(\frac{B}{2}\right) \min \left[\log _{2}\left(1+\frac{P_{R} d_{S R}^{-\alpha}\left|h_{S R}^{\mathrm{Nak}}\right|^{2}}{\sigma_{R}^{2}}\right),\right. \\
\left.\log _{2}\left(1+\frac{P_{s} d_{S D}^{-\alpha}\left|h_{S D}^{\mathrm{Nak}}\right|^{2}}{\sigma_{D}{ }^{2}}+\frac{P_{R} d_{R D}^{-\alpha}\left|h_{R D}^{\mathrm{Nak}}\right|^{2}}{\sigma_{D}^{2}}\right)\right] .
\end{gathered}
$$

\section{B. Outage Analysis}

Outage probability is defined as the probability at node $D$ that the SNR is lower than some predefined threshold and can be written as:

$$
P_{\text {out }}=P\left(\frac{1}{2} \mathrm{I}_{\mathrm{DA}-\mathrm{CC}}^{\xi_{1}, \xi_{2}}<R\right)
$$


where $P_{\text {out }}$ denotes the outage probability at desired node. Putting (7) in (13), outage probability for DA-CC using DF as relaying scheme can be obtained as:

$$
\begin{array}{r}
P_{\text {out }}=P\left(\operatorname { m i n } \left\{\log _{2}\left(1+\mathrm{SNR}_{S R}^{\xi_{2}}\right),\right.\right. \\
\left.\left.\log _{2}\left(1+\mathrm{SNR}_{S D}^{\xi_{1}}+\operatorname{SNR}_{R D}^{\xi_{2}}\right)\right\}<T\right),
\end{array}
$$

where $T=2^{2 R}-1$ and $T$ is some dummy value depending upon receiver sensitivity. Using probability theory, (14) can be written as:

$$
\begin{array}{r}
P_{\text {out }}=\underbrace{P\left[\mathrm{SNR}_{S R}^{\xi_{2}}<T\right]}_{1^{\text {st }} \text { term }}+\underbrace{P\left[\mathrm{SNR}_{S R}^{\xi_{2}}>T\right]}_{2^{\text {nd }} \text { term }} \\
\underbrace{P\left[\mathrm{SNR}_{S D}^{\xi_{1}}+\operatorname{SNR}_{R D}^{\xi_{2}}<T\right]}_{3^{\text {rd }} \text { term }} .
\end{array}
$$

Depending upon $\xi_{1}$ and $\xi_{2}$, three cases for outage probability are possible, which are discussed as follows:

Case 1: In this case, all the channels are considered Rayleigh faded. Putting $\xi_{1}=\xi_{2}=$ Ray in (15), the solution of $1^{\text {st }}$ term can be obtained as:

$$
P\left[\mathrm{SNR}_{S R}^{\text {Ray }}<T\right]=1-e^{-\lambda_{S R} T},
$$

where $\lambda_{S R}=\frac{\sigma_{R}^{2}}{P_{S} d_{S R}^{-\alpha}}$. Solution of $2^{n d}$ term can be obtained with the help of (16). Before finding the $3^{\text {rd }}$ term solution, we need the Probability Density Function (PDF) of the $3^{r d}$ term. Let $3^{r d}$ term be denoted by $\mathrm{Z}$, and its PDF for $\lambda_{S D}=\lambda_{R D}=\lambda$ (rate parameter) can be obtained as:

$$
f_{Z}(z)=\lambda^{2} z e^{-(\lambda) z}
$$

Using (17) in $3^{r d}$ term of (15), the solution of $3^{\text {rd }}$ term can be obtained as :

$$
P\left[\mathrm{SNR}_{S D}^{\mathrm{Ray}}+\mathrm{SNR}_{R D}^{\mathrm{Ray}}<T\right]=\int_{0}^{T} f_{Z}(z) d z .
$$

After integration, $3^{\text {rd }}$ term is obtained as:

$$
P\left[\mathrm{SNR}_{S D}^{\text {Ray }}+\mathrm{SNR}_{R D}^{\mathrm{Ray}}<T\right]=1-e^{-(\lambda) z}(1+\lambda T) .
$$

Putting (16) and (19) in (15), the outage probability for this case can be obtained as:

$$
P_{\text {out }}=\left(1-e^{-(\lambda) z}\right)+\left(e^{-(\lambda) z}\right)\left(1-e^{-(\lambda) z}(1+\lambda T)\right) .
$$

Case 2: In this case, $S-R$ and $R-D$ channels are considered as Nakagami faded, and channel $S-D$ as Rayleigh faded. Putting $\xi_{1}=$ Ray and $\xi_{2}=$ Nak in (15), outage probability in a hybrid channel environment can be obtained as:

$$
\begin{gathered}
P_{\text {out }}=\underbrace{P\left[\mathrm{SNR}_{S R}^{\mathrm{Nak}}<T\right]}_{1^{\text {st }} \text { term }}+\underbrace{P\left[\mathrm{SNR}_{S R}^{\mathrm{Nak}}>T\right]}_{2^{\text {nd }} \text { term }} \\
\underbrace{P\left[\mathrm{SNR}_{S D}^{\mathrm{Ray}}+\mathrm{SNR}_{R D}^{\mathrm{Nak}}<T\right]}_{3^{\text {rd }} \text { term }} .
\end{gathered}
$$

Considering shaping index profile, $m$ of Nakagami faded channel as 2, the SNR equation between $S-R$ link can be shown as:

$$
\mathrm{SNR}_{S R}^{\mathrm{Nak}}=\frac{P_{S} d_{S R}^{-\alpha}\left|h_{S R_{1}}\right|^{2}}{\sigma_{R}^{2}}+\frac{P_{S} d_{S R}^{-\alpha}\left|h_{S R_{2}}\right|^{2}}{\sigma_{R}^{2}} .
$$

Let $\mathrm{SNR}_{S R}^{\mathrm{Nak}}$ be denoted by $X_{1}$, and by using transformation in (22), PDF of $X_{1}$ can be obtained as:

$$
f_{X_{1}}\left(x_{1}\right)=\lambda_{S R}^{2} x_{1} e^{-\lambda_{S R} x_{1}},
$$

where $\lambda_{S R}=\frac{\sigma_{R}^{2}}{P_{S} d_{S R}^{-\alpha}}$. For the same value of $\mathrm{m}, \mathrm{SNR}$ equation between $R-D$ link can be written as:

$$
\mathrm{SNR}_{R D}^{\mathrm{Nak}}=\frac{P_{R} d_{R D}^{-\alpha}\left|h_{R D_{1}}\right|^{2}}{\sigma_{D}^{2}}+\frac{P_{R} d_{R D}^{-\alpha}\left|h_{R D_{2}}\right|^{2}}{\sigma_{D}^{2}} .
$$

Let $\mathrm{SNR}_{R D}^{\mathrm{Nak}}$ be denoted by $X_{2}$. By using transformation in (22), PDF of $X_{2}$ can be obtained as:

$$
f_{X_{2}}\left(x_{2}\right)=\lambda_{R D}^{2} x_{2} e^{-\lambda_{R D} x_{2}},
$$

where $\lambda_{R D}=\frac{\sigma_{D}^{2}}{P_{R} d_{R D}^{-\alpha}}$. Solution of $1^{s t}$ and $2^{n d}$ term can be obtained as:

$$
\begin{array}{r}
P\left(\mathrm{SNR}_{S R}^{\mathrm{Nak}}<T\right)=1-e^{-\lambda T}(1+\lambda T) \\
P\left(\mathrm{SNR}_{S R}^{\mathrm{Nak}}>T\right)=\lambda^{2} T e^{-\lambda T} .
\end{array}
$$

Before finding the solution of $3^{\text {rd }}$ term, we need the PDF of $3^{\text {rd }}$ term. Let $3^{\text {rd }}$ term be denoted by Z, and its PDF for $\lambda_{S D}=\lambda_{R D}=\lambda$, can be obtained as:

$$
f_{Z}(z)=\frac{\lambda^{3} z^{2} e^{-\lambda z}}{2} .
$$

Using (27) in the $3^{\text {rd }}$ term of (21), the solution of the $3^{\text {rd }}$ term can be shown as:

$$
P[Z<T]=\int_{0}^{T} f_{Z}(z) d z .
$$

After integrating, $3^{\text {rd }}$ term is obtained as:

$$
P[Z<T]=0.5\left(-\lambda T e^{-(\lambda) z}(2+T)+2\left(1-e^{-(\lambda) z}\right)\right) .
$$

Putting (26) and (29) in (21), outage probability for this case can be obtained as:

$$
\begin{aligned}
& P_{\text {out }}=\left[1-e^{-\lambda T}(1+\lambda T)\right]+\left[\lambda^{2} T e^{-\lambda T}\right][ \\
& \left.0.5\left(-\lambda T e^{-(\lambda) z}(2+T)+2\left(1-e^{-(\lambda) z}\right)\right)\right] .
\end{aligned}
$$

Case 3: In this case, all the channels are assumed to be Nakagami faded. Putting $\xi_{1}=\xi_{2}=\mathrm{Nak}$ in (15), it can be rewritten as:

$$
\begin{array}{r}
P_{\text {out }}=\underbrace{P\left[\mathrm{SNR}_{S R}^{\mathrm{Nak}}<T\right]}_{1^{\text {st }} \text { term }}+\underbrace{P\left[\mathrm{SNR}_{S R}^{\mathrm{Nak}}>T\right]}_{2^{\text {nd }} \text { term }} \\
\underbrace{P\left[\mathrm{SNR}_{S D}^{\mathrm{Nak}}+\mathrm{SNR}_{R D}^{\mathrm{Nak}}<T\right]}_{3^{\text {rd }} \text { term }} .
\end{array}
$$

The solution of $1^{\text {st }}$ and $2^{\text {nd }}$ term is the same as obtained in (26). Before finding the solution of $3^{\text {rd }}$ term, the PDF of $3^{\text {rd }}$ term is required to be obtained. Let $3^{\text {rd }}$ term be denoted by $\mathrm{Z}$, and its PDF for $\lambda_{S D}=\lambda_{R D}=\lambda$ can be obtained as:

$$
f_{Z}(z)=\frac{\lambda^{4} z^{3} e^{-\lambda z}}{3} .
$$

Using (32) in the $3^{\text {rd }}$ term of (21), the solution of the $3^{\text {rd }}$ term can be obtained as:

$$
P[Z<T]=\int_{0}^{T} f_{Z}(z) d z .
$$


After integration, $3^{r d}$ term can be obtained as:

$$
\begin{aligned}
P[Z<T]= & \frac{1}{6}\left(-\lambda^{3} T^{3} e^{-(\lambda) z}-3 \lambda^{2} T^{2} e^{-(\lambda) z}\right. \\
& \left.-6 \lambda T e^{-(\lambda) z}+6\left(1-e^{-(\lambda) z}\right)\right) .
\end{aligned}
$$

Substituting (26) and (34) in (31), outage probability for this case can be given as:

$$
\begin{aligned}
P_{\text {out }}= & {\left[1-e^{-\lambda T}(1+\lambda T)\right]+\frac{1}{6}\left[\lambda^{2} T e^{-\lambda T}\right]\left(-\lambda^{3} T^{3} e^{-(\lambda) z}\right.} \\
& \left.3 \lambda^{2} T^{2} e^{-(\lambda) z}-6 \lambda T e^{-(\lambda) z}+6\left(1-e^{-(\lambda) z}\right)\right) .
\end{aligned}
$$

\section{Results AND Discussion}

The analysis conducted in the previous section is validated in this section. MATLAB R2013a ${ }^{\circledR}$ is used to perform simulation work. For the current analysis, simulation parameters used are summarized in Table II. The obtained results are evaluated and presented in Fig.6 to 12 . Using DF as a relaying protocol, with the help of outage probability and rate, DA-CC performance has been evaluated.

TABLE II

SIMULATION PARAMETERS

\begin{tabular}{|l|l|}
\hline Simulation Parameters & Values \\
\hline \hline Transmitted Power $\left(P_{t}\right)$ & $10^{-3}$ watt \\
\hline Noise Power $\left(\sigma^{2}\right)$ & $10^{-10}$ watt \\
\hline$\alpha$ & 3 \\
\hline$h_{D}$ & 30 meter \\
\hline$d_{S D}$ & 40 meter \\
\hline Number of Iterations & $10^{5}$ \\
\hline Modulation Scheme & BPSK \\
\hline Shaping Parameter of Nakagami fading $(\mathrm{m})$ & 2 \\
\hline
\end{tabular}

Fig. 6 shows the variation of outage probability with respect to drone height. From this result, it may be noted that outage probability increases with drone height for all the threechannel models for a given source-destination node placement. It may also be noted here that the performance of the hybrid channel model is near to Nakagami faded (case 3). This is due

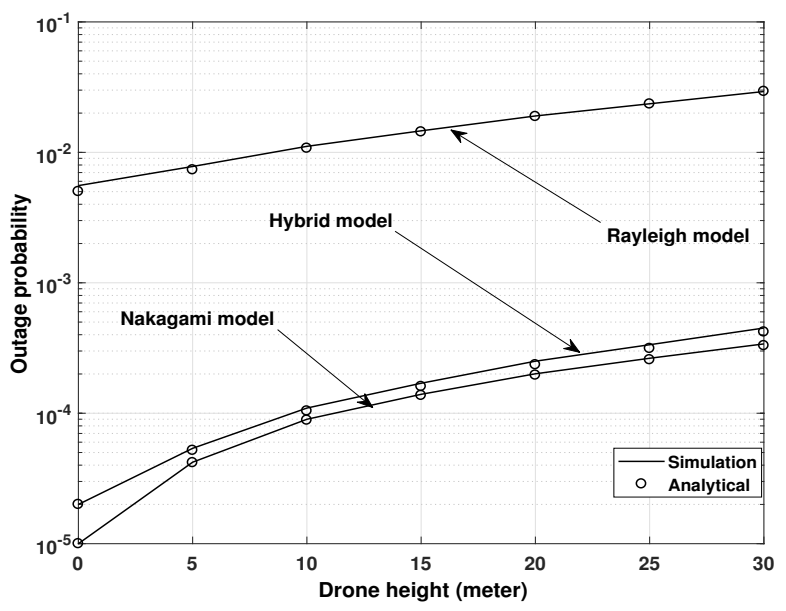

Fig. 6. Outage probability with respect to drone height.

to the fact that as the drone is placed at a greater height, the probability of getting the LoS component increases. While the performance of the Rayleigh faded model (case 1) is the worst among all channel models.

Fig. 7 shows the variation of the outage probability with respect to the horizontal distance between the source-destination pair of nodes under consideration of the different channel conditions. From this result, it is evident that as the horizontal distance between nodes increases, performance goes down for all the three cases. It is also noted here that Nakagami model (case 3) gives better performance in terms of outage probability for a given horizontal distance between nodes and Rayleigh model (case 1) give worse performance.

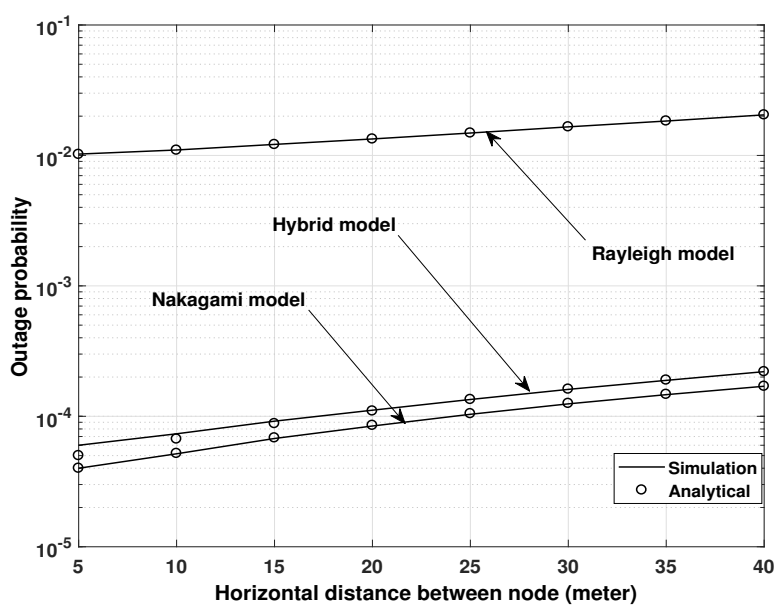

Fig. 7. Outage probability with respect to horizontal distance between $S-D$ pair of nodes.

Fig. 8 shows variation in the outage probability with respect to rate. It may be noted here, for different values of rate the outcome corresponding to the proposed channel model (case 2) lies nearer to the Nakagami faded channel model. This may happen because $\mathrm{A} 2 \mathrm{G}$ characteristics of both the channel models are the same (case 2 and case 3). Decrements in the

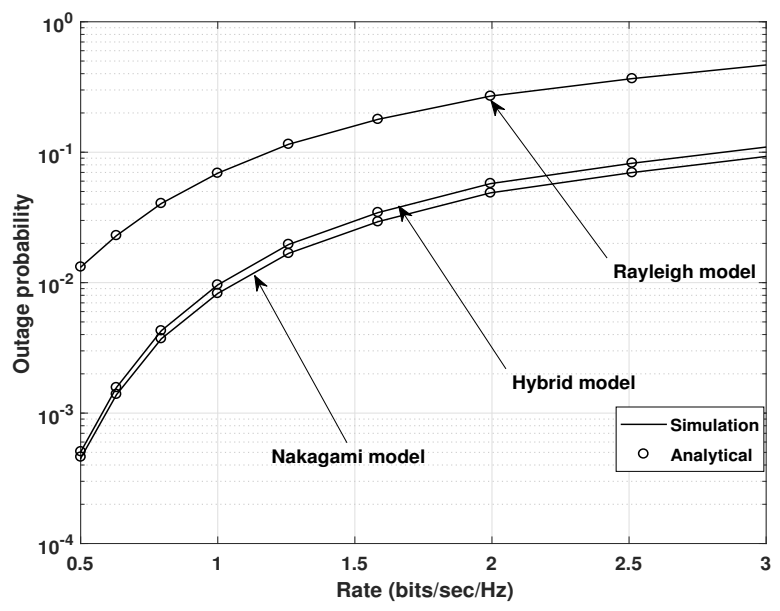

Fig. 8. Outage probability with respect to rate.

performance in terms of outage probability with increment in the required rate for all the three-channel models is due to the 
fact that channel gain continuously goes down for the large values of the required rate.

Variation in outage probability with respect to transmitted power has been demonstrated in Fig.9. It is observed here that the probability of outage decreases as the transmitted power increases. It is also evident from this result that Nakagami model (case 3) provides better results, while Rayleigh model (case 1) provides worse performance in terms of outage probability.

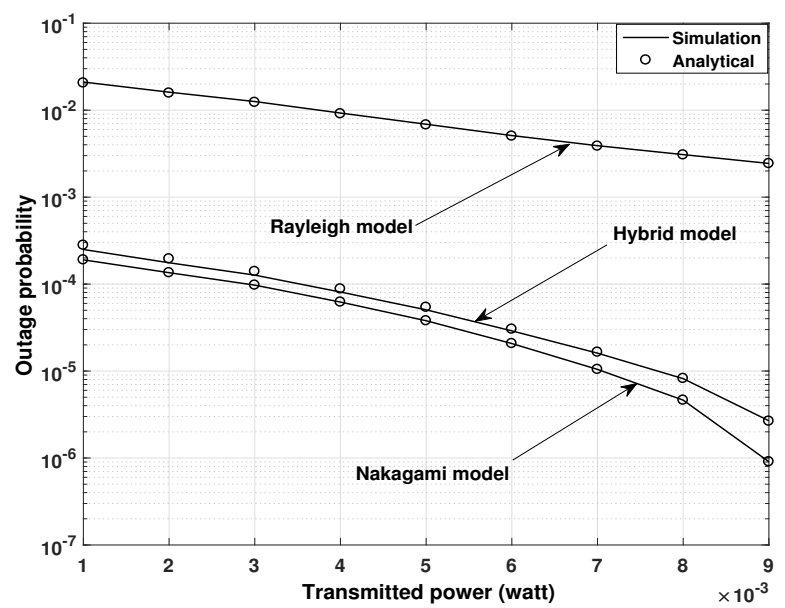

Fig. 9. Outage probability with respect to transmitted power in the presence of different channel conditions.

The behavior of rate with respect to drone height has been demonstrated in Fig. 10. It is noticed that for a given horizontal distance, the rate decreases as drone height increases in all three cases. For a given drone height, the performance of the Nakagami model (case 3) is best, and Rayleigh model (case 1) provides worse performance while the proposed hybrid channel model (case 2) performance lies very close to the Nakagami one.

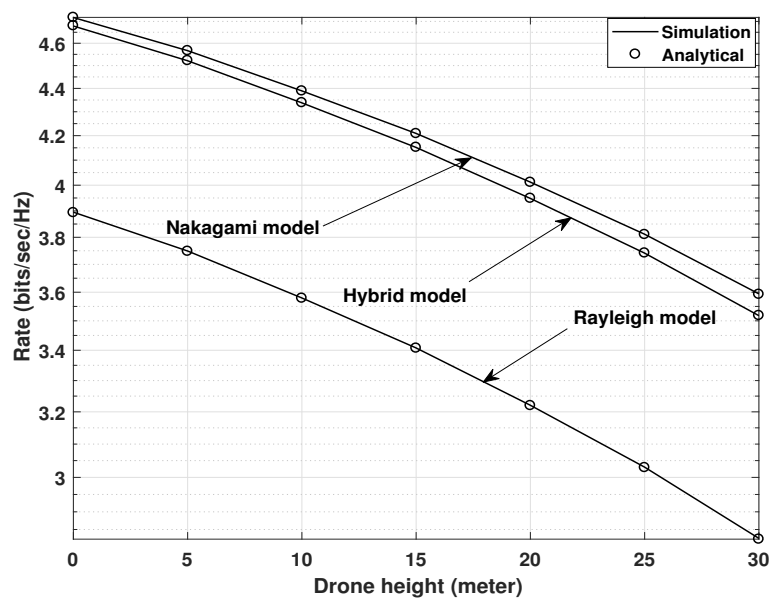

Fig. 10. Rate with respect to drone height.

The behavior of rate with respect to the horizontal distance between $S-D$ pair of nodes is depicted in Fig.11 under

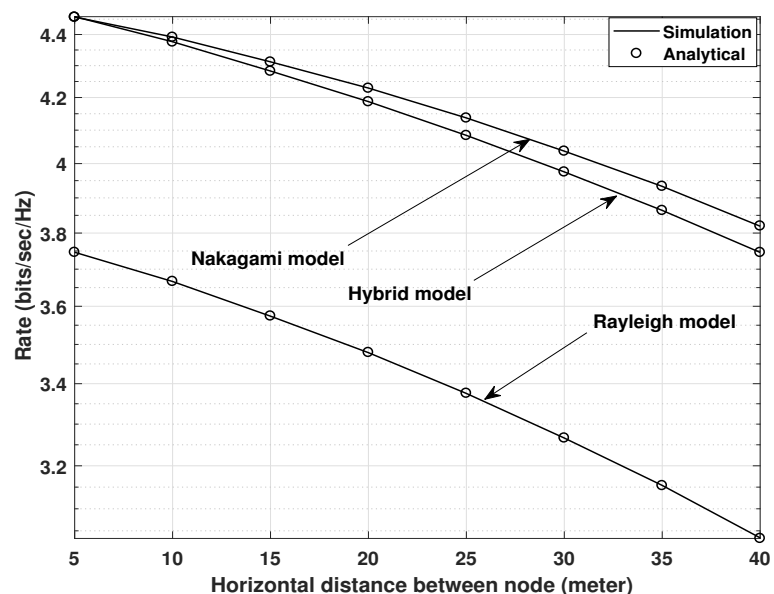

Fig. 11. Rate with respect to the horizontal distance between $S-D$ pair of nodes.

different channel conditions. Here it can be observed that as horizontal distance increases, the rate decreases. It is also evident from this result that Nakagami model (case 3) provides a better rate, while Rayleigh model (case 1) provides the worst rate for the given horizontal distance.

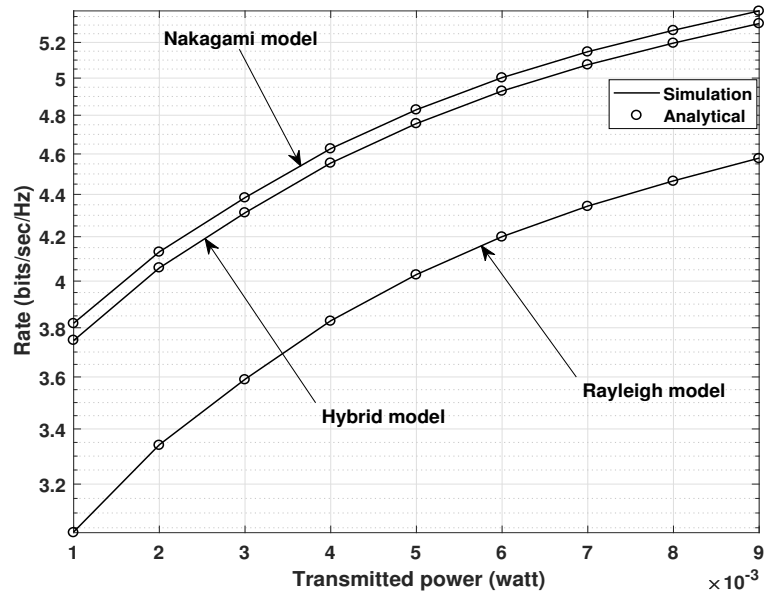

Fig. 12. Rate with respect to transmitted power.

Fig. 12 shows the variation in rate with respect to the transmitted power in the presence of different channel conditions. Here as transmitted power increases, it is observed that rate increases. It is also evident from this result that Nakagami model (case 3) provides a better rate, while Rayleigh model (case 1) provides the worst rate for the given transmitted power.

\section{CONCLUSION}

In this paper, performance analysis of drone-assisted cooperative communication systems is carried out by using cooperation strategy over hybrid fading channels. The above performance analysis is based on DF relaying protocol and MRC. The fact that in an urban area, where ground to ground 
users cannot receive LOS component due to multiple obstacles has been taken into account by considering Rayleigh fading therein. With the usage of the drone as a relay node, an additional $\mathrm{A} 2 \mathrm{G}$ path has been provided to ground users, which provides a better LOS component considered to be Nakagami faded in the proposed system model. With this system model, outage probability and rate equations have been derived. It is shown that the derived results agree with the simulation results, which validates the theoretical analysis. Furthermore, it can be concluded that the performance of the system is good over the hybrid fading channel, which appears to be very close to the system performance when all the channels are Nakagami distributed. Whereas the performance is poor when all the channels are Rayleigh distributed. In the future, we shall extend this work for a general value of shaping parameters (m) which depends on drone height and derive the analytical expression of outage and rate.

\section{REFERENCES}

[1] J. Laneman, D. Tse, and G. Wornell, "Cooperative diversity in wireless networks: Efficient protocols and outage behavior," IEEE Transactions on Information Theory, vol. 50, no. 12, pp. 3062-3080, 2004.

[2] T. Cover and A. Gamal, "Capacity theorems for the relay channel," IEEE Transactions on Information Theory, vol. 25, no. 5, pp. 572-584, 1979

[3] S. Savazzi and U. Spagnolini, "Cooperative fading regions for decode and forward relaying," IEEE Transactions on Information Theory, vol. 54, no. 11, pp. 4908-4924, 2008.

[4] M. Mozaffari, W. Saad, M. Bennis, Y.-H. Nam, and M. Debbah, "A tutorial on uavs for wireless networks: Applications, challenges, and open problems," IEEE Communications Surveys Tutorials, vol. 21, no. 3, pp. 2334-2360, 2019.

[5] N. Zhao, W. Lu, M. Sheng, Y. Chen, J. Tang, F. R. Yu, and K.-K. Wong, "Uav-assisted emergency networks in disasters," IEEE Wireless Communications, vol. 26, no. 1, pp. 45-51, 2019.

[6] P. Kumar, S. Darshi, and S. Shailendra, "Drone assisted device to device cooperative communication for critical environments," IET Communications, vol. 15, no. 7, pp. 957-972, 2021.

[7] M. F. Kader, M. B. Uddin, S. R. Islam, and S. Y. Shin, "Capacity and outage analysis of a dual-hop decode-and-forward relay-aided noma scheme," Digital Signal Processing, vol. 88, pp. 138-148, 2019.

[8] Q. F. Zhou, Y. Li, F. C. M. Lau, and B. Vucetic, "Decode-andforward two-way relaying with network coding and opportunistic relay selection," IEEE Transactions on Communications, vol. 58, no. 11, pp. 3070-3076, 2010.

[9] T. Q. Duong and H.-J. Zepernick, "Hybrid decode-amplify-forward cooperative communications with multiple relays," in 2009 IEEE Wireless Communications and Networking Conference, 2009, pp. 1-6.

[10] _ - "On the performance gain of hybrid decode-amplify-forward cooperative communications," EURASIP Journal on Wireless Communications and Networking, vol. 2009, pp. 1-10, 2009.

[11] S. Ghofrani-Jahromi, A. Zolghadrasli, and M. Neinavaie, "Performance analysis of multihop decode-and-forward relay networks with diversity in nakagami fading channels," AEU-International Journal of Electronics and Communications, vol. 113, p. 152973, 2020.

[12] Y. Lee and M.-H. Tsai, "Performance of decode-and-forward cooperative communications over nakagami- $m$ fading channels," IEEE Transactions on Vehicular Technology, vol. 58, no. 3, pp. 1218-1228, 2009.

[13] Y. Lee, M.-h. Tsai, and S.-i. Sou, "Performance of decode-andforward cooperative communications with multiple dual-hop relays over nakagami-m fading channels," IEEE Transactions on Wireless Communications, vol. 8, no. 6, pp. 2853-2859, 2009.

[14] J. Yao, J. Ye, G. Pan, Y. Xie, and Q. Feng, "Relay selection schemes in threshold df cooperative systems with wireless power transfer," Journal of the Franklin Institute, vol. 357, no. 8, pp. 5091-5109, 2020.

[15] D. Liang, S. X. Ng, and L. Hanzo, "Relay-induced error propagation reduction for decode-and-forward cooperative communications," in 2010 IEEE Global Telecommunications Conference GLOBECOM 2010, 2010, pp. $1-5$.

[16] R. Mohseni and E. Daneshifar, "Robust cooperative beamforming for mimo decode-and-forward one-way relay networks," Physical Communication, vol. 39, p. 100973, 2020.
[17] S. Ikki and M. H. Ahmed, "Performance analysis of cooperative diversity wireless networks over nakagami-m fading channel," IEEE Communications Letters, vol. 11, no. 4, pp. 334-336, 2007.

[18] A. Fotouhi, H. Qiang, M. Ding, M. Hassan, L. G. Giordano, A. GarciaRodriguez, and J. Yuan, "Survey on uav cellular communications: Practical aspects, standardization advancements, regulation, and security challenges," IEEE Communications Surveys Tutorials, vol. 21, no. 4, pp. 3417-3442, 2019.

[19] B. Li, Z. Fei, and Y. Zhang, "Uav communications for 5g and beyond: Recent advances and future trends," IEEE Internet of Things Journal, vol. 6, no. 2, pp. 2241-2263, 2019.

[20] A. Al-Hourani, S. Kandeepan, and S. Lardner, "Optimal lap altitude for maximum coverage," IEEE Wireless Communications Letters, vol. 3, no. 6, pp. 569-572, 2014.

[21] W. Wei, W. Zhang, and M. Yang, "Power allocation scheme for decodeand-forward cooperative communications in rician fading channels," in International Conference on Wireless and Satellite Systems. Springer, 2019, pp. 524-531.

[22] M. Asshad, S. A. Khan, A. Kavak, K. Küçük, and D. L. Msongaleli, "Cooperative communications using relay nodes for next-generation wireless networks with optimal selection techniques: A review," IEEE Transactions on Electrical and Electronic Engineering, vol. 14, no. 5, pp. 658-669, 2019.

[23] J. Zakaria and M. F. M. Salleh, "Performance analysis of cooperative fixed-gain relaying system in severe fading channels," IET Communications, vol. 13, no. 14, pp. 2124-2131, 2019.

[24] P. Kumar, P. Singh, S. Darshi, and S. Shailendra, "Analysis of drone assisted network coded cooperation for next generation wireless network," IEEE Transactions on Mobile Computing, vol. 20, no. 1, pp. 93-103, 2021.

[25] — "Drone assisted network coded co-operation," in TENCON 2019 - 2019 IEEE Region 10 Conference (TENCON), 2019, pp. 1174-1179.

[26] T. Hiraguri, K. Nishimori, I. Shitara, T. Mitsui, T. Shindo, T. Kimura, T. Matsuda, and H. Yoshino, "A cooperative transmission scheme in drone-based networks," IEEE Transactions on Vehicular Technology, vol. 69, no. 3, pp. 2905-2914, 2020.

[27] E. Yanmaz, S. Yahyanejad, B. Rinner, H. Hellwagner, and C. Bettstetter "Drone networks: Communications, coordination, and sensing," Ad Hoc Networks, vol. 68, pp. 1-15, 2018

[28] N. Goel and V. Gupta, "Performance analysis of drone assisted cooperative communication in hybrid channel environment," in 2019 IEEE International Conference on Advanced Networks and Telecommunications Systems (ANTS), 2019, pp. 1-5.

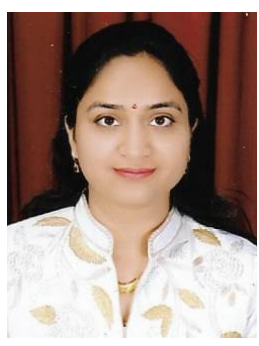

Nikita Goel received her B.Tech degree in Electronics and Communication Engineering (First Division with Honours) from Uttar Pradesh Technical University, Lucknow and M.Tech degree with specialization in Digital Communication (First Division with Honours) from Dr. A.P.J Abdul Kalam Technical University, Lucknow, India. She has 8 years of teaching experience. Currently she is working as Assistant Professor in KIET Group of Institutions, Delhi NCR, Ghaziabad, India, and also Part time Research Scholar in NIT Kurukshetra, Kurukshetra, Haryana. Ms. Nikita Goel research and teaching interests are wireless communications, OFDM, cooperative communications and computer networks.

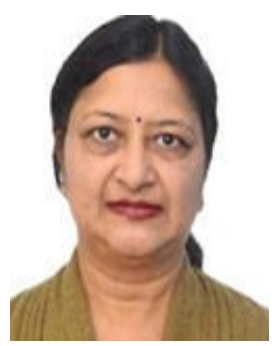

Vrinda Gupta is an Associate Professor in the Department of Electronics and Communication Engineering at NIT Kurukshetra. She is currently heading the Department. She has worked as an Engineering Teacher for more than 27 years. She received Ph.D. and M.Tech. degrees (Electronics and Communication Engineering) from National Institute of Technology Kurukshetra and B.E. degree in Electronics Engineering from Nagpur University. Dr. Vrinda Gupta's research and teaching interests are in the areas of communication networks, and internet of things. She is a member of professional societies, viz. IEEE, IETE, IAENG, and Fellow of IE (I). 